\title{
ON THE ONE-PARTICLE GREEN FUNCTION OF A SYSTEM OF INTERACTING PHONONS
}

\author{
J. J. J. KOKKEDEE
}

Institute for Theoretical Physics of the University of Utrecht, The Netherlands

Received 18 February 1963

In this note we briefly present the results of a calculation, using many-particle perturbation theory, of the one-phonon Green function (or rather its Fourier transform) of an anharmonic crystal to general orde1 in the perturbation potential, i.e., in the phonon-phonon coupling, and for all temperatures well below the melting point. The main results of this work have already been published in two earlier paper $\mathrm{s} 1,2$ ), in which we studied the influence of phonon interactions on the position and broadening of the one-phonon peaks in the inelastic energy spectrum of thermal neutrons, scattered coherently by a single crystal.

In recent years the usefulness of thermodynamic Green functions in describing the properties of manyparticle systems both at absolute zero and at non-vanishing temperatures has been emphasised by many authors 3 ). One way of determining the one-particle Green function is by means of its equation of motion (together with appropriate boundary conditions), which expresses the time derivative of this function in terms of the two-particle Green function. Similarly the equation for the two-particle Green function involves the three-particle Green function, and so on, giving an infinite chain of coupled equations. The exact solution of this chain is, in general, impossible, so that one has to introduce some approximation method to decouple it. Another way of obtaining a useful expression for the one-particle Green function is by direct calculation making use of many-particle perturbation theory and diagram techniques. Of course, this method only applies in the case that the coupling constant, measuring the strength of the interaction, is sufficiently small. For a system of interacting phonons this coupling constant is of the order of $u / d$, which for temperatures well below the melting point is much smaller than one $(u$ is an average atomic displacement at a given temperature, $d$ is the lattice constant). In this case the one-phonon Green functior can be expressed exactly in terms of quantities which involve simple, connected and irreducible diagrams only.

We define the one-phonon Green function as follows

$$
D_{\boldsymbol{q} j j^{\prime}}(t)=\left\langle A_{\boldsymbol{q} j}(0) A_{\boldsymbol{q} j}^{*}(t)\right\rangle_{T}=Z^{-1} \operatorname{Tr}\left\{U_{-\beta} U_{-\mathbf{i} t} A_{\boldsymbol{q} j} U_{\mathbf{i} t} A_{\boldsymbol{q} j}^{*}\right\}
$$

$A_{\boldsymbol{q} j}$ and $A_{\mathbf{q} j}^{*}$ are annihilation and creation operators for the phonon characterised by a wave vector $\boldsymbol{q}$ and a polarisation index $j$, its frequency will be denoted by $\omega_{q j}$;

$$
U_{ \pm \mathrm{i} t} \equiv \exp \left[ \pm \mathrm{i} t\left(H_{\mathrm{O}}+V\right)\right] \quad \text { and } \quad U_{-\beta} \equiv \exp \left[-\beta\left(H_{\mathrm{O}}+V\right)\right] \quad \text { with } \beta=\left(k_{\mathrm{B}} T\right)^{-1} \text {, }
$$

where $H_{\mathrm{O}}$ is the unperturbed (harmonic) Hamiltonian and $V$ is the perturbation (anharmonic potential) ${ }^{\dagger}$. Finally, $Z$ is the partition function. Instead of calculating the function $D_{\boldsymbol{q} j} j,(t)$, we consider its Fourier transform with respect to $t$ separated into a positive and negative frequency part, i.e., we consider the functions

$$
C^{+}\left(\boldsymbol{q} \omega j j^{\prime}\right)=\frac{1}{2 \pi} \int_{0}^{\infty} \mathrm{d} t \mathrm{e}^{-\mathrm{i} \omega t} D_{\boldsymbol{q} j j^{\prime}}(t), \quad C^{-}\left(\boldsymbol{q} \omega j j^{\prime}\right)=\frac{1}{2 \pi} \int_{-\infty}^{0} \mathrm{~d} t \mathrm{e}^{-\mathrm{i} \omega t} D_{\boldsymbol{q} j j^{\prime}}(t) .
$$

It is furthermore advantageous to introduce two similar functions

$$
\widetilde{C}^{+}\left(\boldsymbol{q} \omega j j^{\prime}\right)=\frac{1}{2 \pi} \int_{0}^{\infty} \mathrm{d} t \mathrm{e}^{-\mathrm{i} \omega t} \widetilde{D}_{\boldsymbol{q} j j^{\prime}(t)}, \quad \widetilde{C}^{-}\left(\boldsymbol{q} \omega j j^{\prime}\right)=\frac{1}{2 \pi} \int_{-\infty}^{0} \mathrm{~d} t \mathrm{e}^{-\mathrm{i} \omega t} \widetilde{D}_{\boldsymbol{q} j j^{\prime}}(t),
$$

where $\widetilde{D}_{\boldsymbol{q} j j^{\prime}}(t)$ is defined by

+ We put $\hbar=1$. 


$$
\widetilde{D}_{\boldsymbol{q} j j^{\prime}}(t)=Z^{-1} \operatorname{Tr}\left\{U_{-\beta} U_{-i t} A_{-\boldsymbol{q} j}^{*} U_{\mathbf{i} t} A_{\left.\boldsymbol{q} j^{\prime}\right\}}^{*} .\right.
$$

We have investigated the traces occurring in (1) and (4) by means of diagram techniques. The details of this investigation can be found in ref. 1). A rather complicated diagram analysis leads to the following two coupled equations, analogous to the Dyson equations in field theory, for the functions $C^{ \pm}\left(q \omega j j^{\dagger}\right)$ and $\widetilde{C}^{ \pm}\left(\boldsymbol{q} \omega j j^{\prime}\right)$, valid to general order in the phonon-phonon interaction:

$\mp 2 \pi$ i $C^{ \pm}\left(q \omega j j^{\prime}\right)=\left(\omega_{q j}-\omega \pm i 0\right)^{-1} f_{q j} \delta_{j j^{\prime}}+\left(\omega_{q j}-\omega \pm i 0\right)^{-1} \Theta^{ \pm}\left(q \omega j j^{\prime}\right)$

$$
\pm 2 \pi \mathrm{i} \sum_{j^{n}}\left(\omega_{q j}-\omega \pm i 0\right)^{-1} G^{ \pm}\left(q \omega j j^{n}\right)\left[C^{ \pm}\left(q \omega j^{n} j^{\prime}\right)+\widetilde{C}^{ \pm}\left(q \omega j^{n} j^{\prime}\right)\right],
$$

$\pm 2 \pi$ i $\widetilde{C}^{ \pm}\left(\boldsymbol{q} \omega j j^{\prime}\right)=\left(\omega_{q j}+\omega \mp i 0\right)^{-1} \widetilde{\Theta}^{ \pm}\left(q \omega j j^{\prime}\right)$

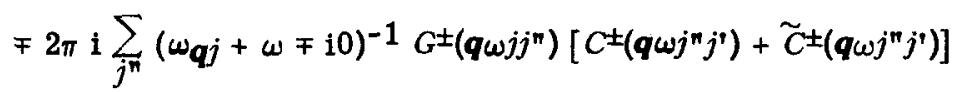

with

$$
\begin{aligned}
G^{ \pm}\left(\boldsymbol{q} \omega j j^{\prime}\right)=\mathbf{i} \int_{0}^{ \pm \infty} \mathrm{d} \tau \mathrm{e}^{-\mathbf{i} \omega \tau} \operatorname{Tr}\left\{U_{-\beta} U_{-\mathbf{i} \tau} V_{\boldsymbol{q} j} U_{\mathbf{i} \tau} V_{\left.\boldsymbol{q} j^{\prime}\right\}_{C, n q}}\right. & \\
+ & +\mathrm{i} \int_{0}^{\mp \infty} \mathrm{d} \tau \mathrm{e}^{\mathbf{i} \omega \tau} \operatorname{Tr}\left\{U_{-\beta} U_{-\mathbf{i} \tau} V_{\boldsymbol{q} j^{\prime}} U_{\mathbf{i} \tau} V_{\boldsymbol{q} j}\right\}_{C, n q}+\operatorname{Tr}\left\{U_{-\beta} V_{\boldsymbol{q} j j^{\prime}}\right\}_{C, n q},
\end{aligned}
$$

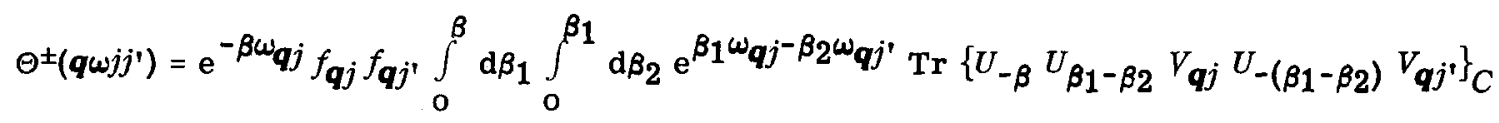

$+\mathrm{e}^{-\beta \omega_{\boldsymbol{q} j}} f_{\boldsymbol{q} j} f_{\boldsymbol{q} j^{*}} \int_{0}^{\beta} \mathrm{d} \beta_{1} \int_{0}^{\beta_{1}} \mathrm{~d} \beta_{2} \mathrm{e}^{-\beta_{1} \omega_{\boldsymbol{q}} j^{+}+\beta_{2} \omega_{\boldsymbol{q} j}} \operatorname{Tr}\left\{U_{-\beta} U_{\beta_{1}-\beta_{2}} V_{\boldsymbol{q} j^{\prime}} U_{-\left(\beta_{1}-\beta_{2}\right)} V_{\boldsymbol{q} j}\right\}_{C}$

$+\left(\omega_{\boldsymbol{q} j}-\omega_{\boldsymbol{q} j^{\prime}}\right) f_{\boldsymbol{q} j} f_{\boldsymbol{q} j^{\prime}}\left(\mathrm{e}^{-\beta \omega_{\boldsymbol{q} j}}-\mathrm{e}^{\left.-\beta \omega_{\boldsymbol{q}} j^{r}\right)} \operatorname{Tr}\left\{U_{-\beta} V_{\boldsymbol{q} j j^{\prime}}\right\}_{C, n \boldsymbol{q}}\right.$

$-i \sum_{j^{m}} \int_{0}^{\beta} \mathrm{d} \beta_{1} \int_{0}^{ \pm \infty} \mathrm{d} \tau \operatorname{Tr}\left\{U_{-\beta} U_{\beta_{1}} U_{\mathbf{i} \tau} V_{\boldsymbol{q} j^{m}} U_{-\beta_{1}} U_{-\mathbf{i} \tau} V_{\boldsymbol{q} j}\right\}_{C, n q} \mathrm{e}^{-\mathbf{i} \omega \tau} \operatorname{Tr}\left\{U_{-\beta} U_{\beta_{1}}\left(A_{\boldsymbol{q} j^{m}}+A_{-\boldsymbol{q} j^{m}}^{*}\right) U_{-\beta_{1}} A_{\boldsymbol{q} j^{*}}^{*}\right\}_{C}$,

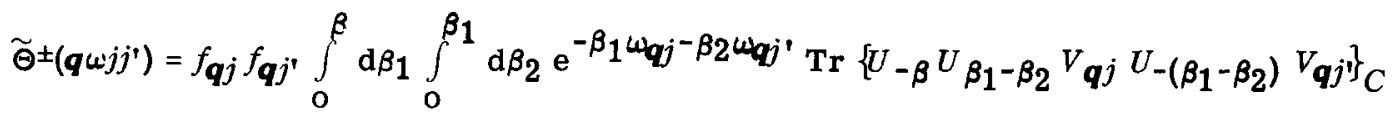

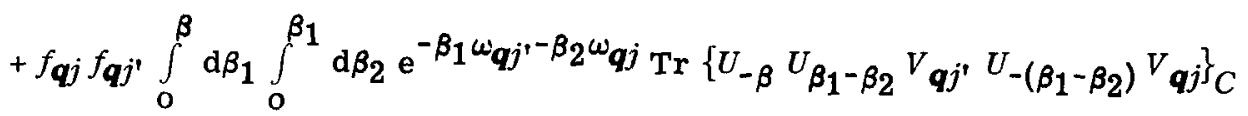

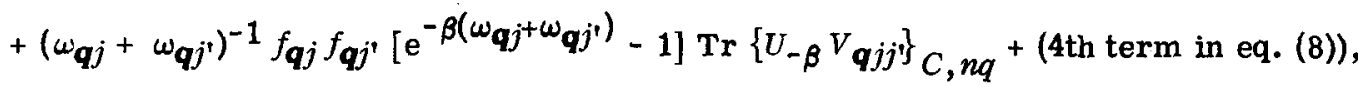

$$
\begin{aligned}
& f_{\boldsymbol{q} j}=\left[1-\exp \left(-\beta \omega_{\boldsymbol{q} j}\right)\right]^{-1} .
\end{aligned}
$$

In eqs. (5) and (6) either the upper sign has to be taken or the lower sign. The index pair $q j$ at the operator $V$ in the traces in (7), (8) and (9) indicates that $V$ contains a creation operator for the phonon $q j$ (or

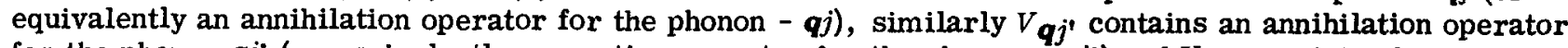
for the phonon $q j^{\prime}$ (or equivalently a creation operator for the phonon $-q j^{\prime}$ ) and $V_{q} j^{\prime}$ contains both. The quantities $G^{ \pm}, \Theta^{ \pm}$and $\widetilde{\Theta}^{ \pm}$, which depend on temperature, are expressed in terms of connected diagrams (subscript $C$ ) or proper connected (irreducible) diagrams (subscript $C, n q$; for details we must refer to ref. 1)).

If we neglect the dependence on the polarisation indices for convenience, the matrix equations (5) and (6) become algebraic equations for the functions $C^{ \pm}(q \omega)$ and $\widetilde{C}^{ \pm}(q \omega)$ which can be solved easily. One finds for $C^{ \pm}(q \omega)$ : 


$$
2 \pi \text { i } C^{ \pm}(\boldsymbol{q} \omega)=\frac{ \pm\left[f_{\boldsymbol{q}}+\Theta^{ \pm}(\boldsymbol{q} \omega)\right]\left[\omega+\omega_{\boldsymbol{q}}+G^{ \pm}(\boldsymbol{q} \omega)\right] \pm G^{ \pm}(\boldsymbol{q} \omega) \Theta^{ \pm}(\boldsymbol{q} \omega)}{\left[\omega-\omega_{\boldsymbol{q}}-G^{ \pm}(\boldsymbol{q} \omega)\right]\left[\omega+\omega_{\boldsymbol{q}}+G^{ \pm}(\boldsymbol{q} \omega)\right]+\left[G^{ \pm}(\boldsymbol{q} \omega)\right]^{2}}
$$

This equation has a similar structure as the one derived by Beliaev ${ }^{4)}$ and by Hugenholtz and Pines 5 ) for a boson gas in its ground state $\left(T=0^{\circ} \mathrm{K}\right)$. It can also be written as follows

$$
2 \pi \text { i } C^{ \pm}(\boldsymbol{q} \omega)=\frac{ \pm\left[f_{\boldsymbol{q}}+\Theta^{ \pm}(\boldsymbol{q} \omega)\right]\left[\omega+\omega_{\boldsymbol{q}}+G^{ \pm}(\boldsymbol{q} \omega)\right] \pm G^{ \pm}(\boldsymbol{q} \omega) \widetilde{\Theta}^{ \pm}(\boldsymbol{q} \omega)}{\left[\omega-\omega_{\boldsymbol{q}}-\Delta(\boldsymbol{q} \omega) \mp \mathrm{i} \Gamma(\boldsymbol{q} \omega)\right]\left[\omega+\omega_{\boldsymbol{q}}+\Delta(\boldsymbol{q} \omega) \pm \mathrm{i} \Gamma(\boldsymbol{q} \omega)\right]}
$$

with the abbreviations

$$
\begin{aligned}
& \Delta(\boldsymbol{q} \omega)=\operatorname{Re}\left[\omega_{\boldsymbol{q}}\left\{1+\left(2 G^{+}(\boldsymbol{q} \omega) / \omega_{\boldsymbol{q}}\right)\right\}^{\frac{1}{2}}-\omega_{\boldsymbol{q}}\right], \\
& \Gamma(\boldsymbol{q} \omega)=\operatorname{Im}\left[\omega_{\boldsymbol{q}}\left\{1+\left(2 G^{+}(\boldsymbol{q} \omega) / \omega_{\boldsymbol{q}}\right)\right\}^{\frac{1}{2}}-\omega_{\boldsymbol{q}}\right] .
\end{aligned}
$$

To lowest order in the coupling, i.e., the order $(u / d)^{2}$, one has

$$
2 \pi \mathrm{i} C f_{(1)}(\boldsymbol{q} \omega)= \pm\left[f_{\boldsymbol{q}}+\Theta_{(1)}^{ \pm}\left(\boldsymbol{q} \omega_{\boldsymbol{q}}\right)\right] /\left[\omega-\omega_{\boldsymbol{q}}-\Delta_{(1)}\left(\boldsymbol{q} \omega_{\boldsymbol{q}}\right) \mp \mathrm{i} \Gamma_{(1)}\left(\boldsymbol{q} \omega_{\boldsymbol{q}}\right)\right] \text {. }
$$

It will be clear from this equation that $\Delta_{(1)}=\operatorname{Re} G_{(1)}^{+}$and $\Gamma(1)=\operatorname{Im} G_{(1)}^{+}$describe to lowest order the shift and spread of the phonon energy due to phonon-phonon interaction, corresponding to a self-energy and finite lifetime of the phonon ${ }^{\dagger}$. It has been proved possible to measure these effects by means of inelastic scattering of slow neutrons 6). Explicit expressions for $\Delta(1)$ and $\Gamma(1)$ in terms of the parameters of the lattice and as functions of the temperature have been derived in ref. 1). In the same way the quantity $\Theta_{(1)}^{ \pm}$can be obtained from the lowest order diagrams contributing to (8) using the rules for calculating diagrams explained in ref. 1). From (11) - (14) we see that also to general order the shift and width of the energy states of the phonons are determined by the complex proper self-energy function $G^{ \pm}(\boldsymbol{q} \omega)$, or better $G^{ \pm}\left(\boldsymbol{q} \omega j j^{\prime}\right)$. However, in higher order (to order $(u / d)^{n}$ with $n>2$ ) the shift and width are no longer simply the hermitian and anti-hermitian part of this quantity (compare (13) and (14)); the effects of shifting and broadening are interdependent in the general case. Observe that the important $G$-function, defined by (7), is completely expressed in terms of proper, connected diagrams. Therefore an expansion of $G^{ \pm}\left(q \omega_{j} j j^{\prime}\right)$ in powers of the coupling constant $u / d$ is expected to converge rapidly for all values of $\omega$, while for any particular order only a finite number of essentially different diagrams need to be calculated. We should remark that in order to be able to consider the phonon as a quasiparticle also in higher order in the couplin the following condition must be satisfied: the width of the energy state of a phonon must be small compared to the energy itself, independent of the strength of the coupling. We expect this to be true for temperatures well below the melting point and/or values of the phonon wave vector which are sufficiently small.

For given $j$ and $j^{\prime}$ there exists a simple relation between the real and imaginary part of the proper sel energy function $G^{ \pm}\left(q \omega j j^{\prime}\right)$, defined by eq. (7). It can be shown that the traces in the first two terms on the right hand side of (7) have the following property: they are bounded for all values of $\tau$ and go to zero for $|\tau| \gg \omega_{D}^{-1}$, where $\omega_{D}$ is the maximum phonon frequency. According to a theorem due to Titchmarsh 7 ) well known in the theory of dispersion relations, the following relations hold (note that the third term in (7) is real and write $\left.G^{ \pm}\left(\boldsymbol{q} \omega j j^{\prime}\right)=\operatorname{Re} G^{+}\left(\boldsymbol{q} \omega j j^{\prime}\right) \pm i \operatorname{Im} G^{+}\left(\boldsymbol{q} \omega i j^{\prime}\right)\right)$ :

$$
\begin{aligned}
& \operatorname{Im} G^{+}\left(\boldsymbol{q} \omega j j^{\prime}\right)=\frac{1}{\pi} P \int_{-\infty}^{\infty} \frac{\operatorname{Re} G^{+}\left(\boldsymbol{q} \omega^{\prime} j j^{\prime}\right)-F\left(\boldsymbol{q} j j^{\prime}\right)}{\omega^{\prime}-\omega} \mathrm{d} \omega^{\prime}, \\
& \operatorname{Re} G^{+}\left(\boldsymbol{q} \omega_{j j} j^{\prime}\right)-F\left(\boldsymbol{q} j j^{\prime}\right)=-\frac{1}{\pi} P \int_{-\infty}^{\infty} \frac{\operatorname{Im} G^{+}\left(\boldsymbol{q} \omega^{\prime} j j^{\prime}\right)}{\omega^{\prime}-\omega} \mathrm{d} \omega^{\prime} .
\end{aligned}
$$

In these equations $F\left(q j j^{\prime}\right)$ represents the third $\omega$-independent term on the right hand side of (7). Each of the first two terms of eq. (7) separately satisfies a similar dispersion relation. Eqs. (16) and (17), whic] hold generally, may be useful in numerical computations of phonon widths and shifts; in this connection they were written down for the lowest order contributions to $G^{+}\left(g \omega j j^{\prime}\right)$, i.e., for $\Delta(1)$ and for $\Gamma(1)$, by $\mathbf{M} \varepsilon$ radudin et al. 8 ).

+ Note that the unperturbed energies $v_{q}$ are themselves temperature dependent due to thermal expansion (compare re 
We finally remark that the above theory can be extended to the case of conducting crystals, where in addition to phonon-phonon coupling there is an interaction between phonons and conduction electrons 2).

This work is part of the research program of the "Stichting voor Fundamenteel Onderzoek der Materie", which is financially supported by the Netherlands Organisation for pure Scientific Research (Z.W.O.).

\section{References}

1) J.J.J. Kokkedee, Physica 28 (1962) 374.

2) J.J. J.Kokkedee, Physica 28 (1962) 893.

3) For surveys see: V. L. Bonch-Bruevich and S.V. Tyablikov, The Green function method in statistical mechanics (North-Holland Publishing Company, Amsterdam, 1962).

L. P. Kadanoff and G. Baym, Quantum statistical mechanics (W. A. Benjamin, New York, 1962).

4) S. T. Beliaev, Soviet Phys. JETP 7 (1958) 289 .
5) N.M. Hugenholtz and D. Pines, Phys. Rev. 116 (1959) 489.

6) K. E. Larsson et al., Ark. Fysik 17 (1960) 369. B.N. Brockhouse et al., in: Inelastic scattering of neutrons in solids and liquids (International Atomic Energy Agency, Vienna, 1961).

7) E.C. Titchmarsh, Theory of Fourier integrals, Ch. $\mathrm{V}$ (Oxford University Press, 1948).

8) A.A.Maradudin, A.E. Fein and G.H. Vineyard, Phys. Stat. Sol. 2 (1962) 1479.

\section{HIGHER SYMMETRIES AND CURRENT CONSERVATION}

\section{Y. NE'EMAN}

Israel Atomic Energy Commission Laboratories, Rehovot:1 and Department of Physics, Tel-Aviv University

Received 14 February 1963

In his rapporteur's review on group-theoretical methods at the 1962 CERN "Rochester" Conference, D'Espagnat 1) has noted a paradox, appearing in the higher symmetry approach, upon imposition of conditions derived from the weak interactions. None of the three prevailing models of strong interaction higher symmetry contain both $|\Delta T|=\frac{1}{2}$ and $|\Delta T|=\frac{3}{2}$ components in their regular (adjoint) representations (this operates in the space of the infinitesimal algebra of the appropriate group). The Sakata 2) and the Gell-Mann Ne'eman 3) SU(3) structures have an octet in this representation (fig. 1) with no $|\Delta T|=\frac{3}{2}$, whereas the Behrends Sirlin 4) G(2) model has a 14-

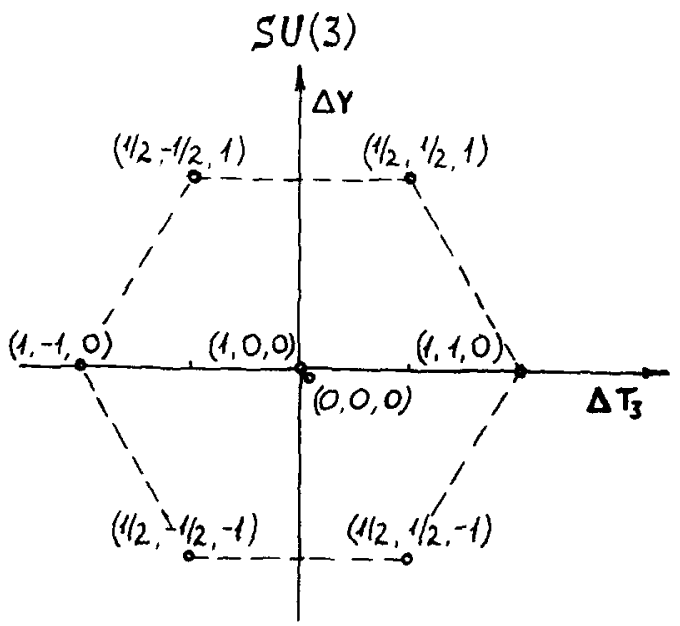

Fig. 1 . component "star of David" representation (fig. 2) with no $|\Delta T|=\frac{1}{2}$. Experimental evidence as exhibited in the hadrons' (strongly-interacting particles 5) decays requires both values to appear in the

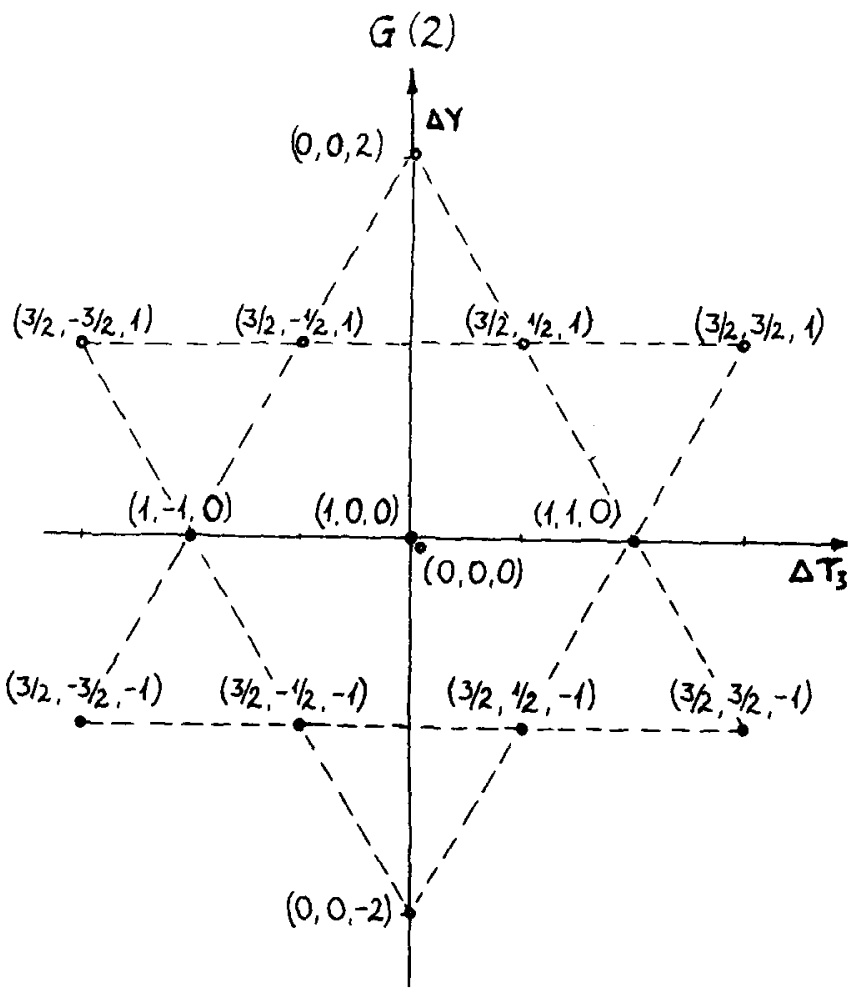

Fig. 2. 